\title{
Potential Biomarkers for Post-Stroke Cognitive Impairment: A Systematic Review and Meta-Analysis
}

\author{
Ka Young Kim ${ }^{1,2} \mathbb{D}$, Ki Young Shin ${ }^{3, *}$ and Keun-A Chang $2,4,5, * \mathbb{D}$
}

Citation: Kim, K.Y.; Shin, K.Y.; Chang, K.-A. Potential Biomarkers for Post-Stroke Cognitive Impairment: A Systematic Review and Meta-Analysis. Int. J. Mol. Sci. 2022, 23, 602. https://doi.org/ $10.3390 /$ ijms23020602

Academic Editors: Teresa Gasull and Adria Arboix

Received: 11 November 2021

Accepted: 23 December 2021

Published: 6 January 2022

Publisher's Note: MDPI stays neutral with regard to jurisdictional claims in published maps and institutional affiliations.

Copyright: (C) 2022 by the authors. Licensee MDPI, Basel, Switzerland. This article is an open access article distributed under the terms and conditions of the Creative Commons Attribution (CC BY) license (https:// creativecommons.org/licenses/by/ $4.0 /)$.

\author{
Department of Nursing, College of Nursing, Gachon University, Incheon 21936, Korea; kykim@gachon.ac.kr \\ Neuroscience Research Institute, Gachon University, Incheon 21565, Korea \\ Bio-MAX Institute, Seoul National University, Seoul 08826, Korea \\ Department of Pharmacology, College of Medicine, Gachon University, Incheon 21936, Korea \\ 5 Neuroscience of Health Sciences and Technology, Gachon Advanced Institute for Health Sciences and \\ Technology, Gachon University, Incheon 21936, Korea \\ * Correspondence: newsky73@gmail.com (K.Y.S.); keuna705@gachon.ac.kr (K.-A.C.)
}

\begin{abstract}
Stroke is a primary debilitating disease in adults, occurring in 15 million individuals each year and causing high mortality and disability rates. The latest estimate revealed that stroke is currently the second leading cause of death worldwide. Post-stroke cognitive impairment (PSCI), one of the major complications after stroke, is frequently underdiagnosed. However, stroke has been reported to increase the risk of cognitive impairment by at least five to eight times. In recent decades, peripheral blood molecular biomarkers for stroke have emerged as diagnostic, prognostic, and therapeutic targets. In this study, we aimed to evaluate some blood-derived proteins for stroke, especially related to brain damage and cognitive impairments, by conducting a systematic review and meta-analysis and discussing the possibility of these proteins as biomarkers for PSCI. Articles published before 26 July 2021 were searched in PubMed, Embase, the Web of Science, and the Cochrane Library to identify all relevant studies reporting blood biomarkers in patients with stroke. Among 1820 articles, 40 were finally identified for this study. We meta-analyzed eight peripheral biomarker candidates: homocysteine (Hcy), high-density lipoprotein cholesterol (HDL-C), C-reactive protein (CRP), low-density lipoprotein cholesterol (LDL-C), total cholesterol (TC), triglyceride (TG), uric acid, and glycated hemoglobin (HbA1c). The Hcy, CRP, TC, and LDL-C levels were significantly higher in patients with PSCI than in the non-PSCI group; however, the HDL-C, TG, uric acid, and $\mathrm{HbA1c}$ levels were not different between the two groups. Based on our findings, we suggest the Hcy, CRP, TC, and LDL-C as possible biomarkers in patients with post-stroke cognitive impairment. Thus, certain blood proteins could be suggested as effective biomarkers for PSCI.
\end{abstract}

Keywords: stroke; dementia; cognitive impairment; post-stroke cognitive impairment; blood biomarker

\section{Introduction}

Stroke occurs in 15 million individuals each year, causing high mortality and disability rates. The latest estimate revealed that stroke is the second leading cause of death worldwide [1,2]. Most strokes are ischemic, owing to the presence of a reduced blood flow, generally resulting from arterial occlusion. The remaining $10-40 \%$ of stroke presentations are hemorrhagic, depending on regional epidemiology and resulting from the rupture of the cerebral arteries [3,4]. Structural damage to the brain in patients with stroke occurs because of both ischemia and hemorrhage [5-7]. As a result, even minor stroke affects daily functions, executive functions, and cognition, consequently affecting patients' activity performance, quality of life, and ability to return to work $[8,9]$.

In particular, the cognitive domains involved in the development of dementia after stroke may vary depending on the stroke characteristics, such as stroke type, volume, number, location, and severity $[10,11]$. (1) Important critical locations include the dominant hemisphere and lesions affecting the prefrontal-subcortical circuit that mediates executive 
dysfunction [12,13]. (2) Frontal lobe functions comprising processing speed, reaction time, working memory, and executive task measures are most commonly affected [14]. (3) A single large cortico-subcortical brain ischemic lesion may present with acute cognitive deterioration if located in an area that is functionally critical for cognition [15]. (4) Strategic infarction dementia may be caused by damage to the components of the Papez (hippocampal memory loop) [16] or Yokovlev circuits [11].

Generally, stroke diagnosis depends crucially on neuroimaging; computed tomography remains an essential component of stroke management, although it is not always available [17]. Over the last decades, molecular biomarkers for stroke have gained the attention of clinicians around the world owing to their broad application in facilitating diagnosis, characterizing clinical size and severity, estimating long-term prognoses, and selecting an appropriate treatment option $[18,19]$. The National Institutes of Health Biomarkers Definitions Working Group proposed a new definition of biomarkers in 2001: "a characteristic that is objectively measured and evaluated as an indicator of normal biological processes, pathogenic processes, or pharmacologic responses to a therapeutic intervention" [20,21]. Thus, biomarkers are beneficial for patients, caregivers, and clinicians for: (a) planning subsequent clinical pathways and goal setting; and (b) identifying whom and when to target and, in some instances, at which level to use, along with interventions for promoting stroke recovery [22]. The application of these biomarkers can improve risk stratification and therefore guide the implementation of tailored treatment modalities [23].

Unlike general stroke, post-stroke cognitive impairment (PSCI), one of the major complications after stroke, is frequently underdiagnosed, as it may be overlooked in the presence of other distressing signs (e.g., motor or visual symptoms). Consequently, the cognitive impact of acute stroke is often underestimated [24,25]. However, stroke has been reported to increase the risk of cognitive impairment by at least five to eight times [26]. To diagnose PSCI, in fact, neuroimaging features of computed tomography (CT) or magnetic resonance imaging (MRI) such as functional MRI (fMRI) and diffusion tensor imaging (DTI) have been used $[27,28]$ and cognitive assessments such as the Information Questionnaire on Cognitive Decline in the Elderly (IQCODE), the Mini-Mental State Examination (MMSE), and the National Institute of Neurological Disease and Stroke (NINDS) battery have been applied [29].

Therefore, we aimed to evaluate some blood-derived proteins, especially those related to brain damage and cognitive impairments, by conducting a systematic review and metaanalysis and discussing the possibility of these proteins as biomarkers for PSCI.

\section{Results}

\subsection{Characteristics of the Included Studies Reporting Potential Biomarkers for PSCI}

We identified 2240 studies including 673 from PubMed, 1081 from Embase, 444 from the Web of Science, and 42 from the Cochrane Library (Figure 1). Duplicates were then excluded, yielding a total of 1820 studies. Thereafter, 84 studies were assessed for eligibility based on their title and abstract after excluding the following studies: (1) studies that did not investigate potential blood biomarkers for evaluating cognitive function after stroke, (2) studies that used cell or animal models, and (3) commentaries, letters, editorials, conference abstracts, or reviews. After a full-text review, 40 articles were finally included in this study.

Table 1 shows the selected studies that reported potential blood biomarkers for PSCI. The included studies were published between 2004 and 2021. The countries of patients were Lithuania, Argentina, Japan, Taiwan, the USA, France, China, Canada, Israel, Poland, the Russian Federation, Sweden, and Turkey. The types of groups used included poststroke without dementia and post-stroke dementia groups, drug or related molecular concentration groups, stroke progression groups, and Mini-Mental State Examination (MMSE) level groups. The sample size of the case and control groups ranged from 8 to 1029 , dividing male and female patients and presenting the mean age in each group. The outcome measurement tools used for evaluating cognitive impairment included the MMSE, 
Montreal Cognitive Assessment, and clinical dementia rating. Furthermore, the sample specimens used were the plasma or serum. Potential blood biomarkers were identified in the selected studies.



Figure 1. Flow diagram of the study selection.

Table 1. Summary of the 40 selected studies reporting potential biomarkers for PSCI.

\begin{tabular}{|c|c|c|c|c|c|c|c|}
\hline $\begin{array}{l}\text { Author and } \\
\text { Year }\end{array}$ & Country & Study Groups & $\begin{array}{c}\text { Sample } \\
\text { Size }(M / F)\end{array}$ & Age (y) & $\begin{array}{c}\text { Outcome } \\
\text { Measurement Tool }\end{array}$ & Specimen & Potential Biomarkers \\
\hline $\begin{array}{l}\text { Bunevicius } \\
\text { et al., } 2015\end{array}$ & Lithuania & $\begin{array}{l}\text { Acute ischemic stroke } \\
\text { Hemorrhagic stroke }\end{array}$ & $53 / 25$ & 72 & MMSE & Serum & NT-proBNP, IL-6, hs-CRP \\
\hline \multirow{3}{*}{$\begin{array}{l}\text { Casas et al., } \\
2017\end{array}$} & \multirow[t]{2}{*}{ Argentina } & Control & $20 / 20$ & $70 \pm 3 / 77 \pm 1$ & \multirow[t]{2}{*}{ MoCA } & \multirow[t]{2}{*}{ Plasma } & \multirow[t]{2}{*}{$\mathrm{BDNF}, \mathrm{NO}^{-2}$} \\
\hline & & Acute ischemic stroke & $20 / 20$ & $72 \pm 4 / 83 \pm 2$ & & & \\
\hline & \multirow[t]{4}{*}{ Japan } & Control & $88 / 104$ & $62.2 \pm 4.4$ & \multirow{4}{*}{$\begin{array}{l}\text { The dementia } \\
\text { status was } \\
\text { classified into six } \\
\text { ranks. }\end{array}$} & \multirow[t]{4}{*}{ Serum } & \multirow[t]{4}{*}{ hs-CRP } \\
\hline \multirow[t]{3}{*}{$\begin{array}{l}\text { Chei et al., } \\
2014\end{array}$} & & $\begin{array}{l}\text { Dementia with a } \\
\text { history of stroke }\end{array}$ & $44 / 52$ & $62.4 \pm 4.3$ & & & \\
\hline & & Control & $98 / 260$ & $62.8 \pm 5.6$ & & & \\
\hline & & $\begin{array}{l}\text { Dementia without a } \\
\text { history of stroke }\end{array}$ & $49 / 130$ & $63.1 \pm 5.6$ & & & \\
\hline \multirow{2}{*}{$\begin{array}{l}\text { Chen et al., } \\
\text { 2019a }\end{array}$} & \multirow[t]{2}{*}{ Taiwan } & $\begin{array}{c}\text { Post-stroke without } \\
\text { dementia }\end{array}$ & $56 / 31$ & $62.98 \pm 9.23$ & \multirow[t]{2}{*}{ CDR } & \multirow[t]{2}{*}{ Plasma } & \multirow[t]{2}{*}{ BChE } \\
\hline & & Post-stroke dementia & $18 / 12$ & $73.20 \pm 8.68$ & & & \\
\hline \multirow{2}{*}{$\begin{array}{l}\text { Chen et al., } \\
\text { 2019b }\end{array}$} & \multirow[t]{2}{*}{ Taiwan } & $\begin{array}{c}\text { Post-stroke without } \\
\text { dementia }\end{array}$ & $41 / 12$ & $61.7 \pm 8.95$ & \multirow[t]{2}{*}{ MMSE } & \multirow[t]{2}{*}{ Plasma } & \multirow[t]{2}{*}{ D-amino acid oxidase } \\
\hline & & Post-stroke dementia & $11 / 9$ & $69.35 \pm 7.24$ & & & \\
\hline \multirow[t]{2}{*}{$\begin{array}{l}\text { Choi et al., } \\
2020\end{array}$} & \multirow[t]{2}{*}{ USA } & $\begin{array}{l}\text { Acute ischemic stroke } \\
\text { alone } \\
\text { Acute ischemic stroke }\end{array}$ & $27 / 8$ & $64.5 \pm 14.1$ & & \multirow[t]{2}{*}{ Serum } & \multirow[t]{2}{*}{$\begin{array}{l}\text { IL-6, CRP, complement } \\
\text { component 3, S100B }\end{array}$} \\
\hline & & $\begin{array}{c}\text { Acute iscnemic stroke } \\
\text { and underlying } \\
\text { dementia }\end{array}$ & $5 / 3$ & $85.8 \pm 9.6$ & & & \\
\hline \multirow[t]{2}{*}{$\begin{array}{l}\text { Cogo et al., } \\
2021\end{array}$} & \multirow[t]{2}{*}{ France } & $\begin{array}{l}\text { Post-stroke cognitive } \\
\text { decline }\end{array}$ & $6 / 4$ & $64.7 \pm 13.3$ & \multirow[t]{2}{*}{ MMSE } & \multirow[t]{2}{*}{ Serum } & \multirow{2}{*}{$\begin{array}{l}\text { Quinolinic acid, quinolinic } \\
\text { acid/kynurenic acid ratio, } \\
\text { tryptophan, kynurenine, } \\
\text { kynurenic acid, } \\
\text { kynurenine/tryptophan } \\
\text { ratio, indoleamine } \\
\text { 2,3-dioxygenase }\end{array}$} \\
\hline & & $\begin{array}{c}\text { Post-stroke cognitive } \\
\text { decline }\end{array}$ & $8 / 5$ & $69.4 \pm 17.8$ & & & \\
\hline $\begin{array}{l}\text { El Hussini } \\
\text { et al., } 2020\end{array}$ & USA & $\begin{array}{l}\text { Small-vessel-type } \\
\text { stroke }\end{array}$ & $9 / 13$ & $56.5(49.5-62.0)$ & $\begin{array}{l}\text { A standardized } \\
\text { battery of } \\
\text { neuropsychological } \\
\text { tests }\end{array}$ & Plasma & $\begin{array}{c}\text { VCAM-1, IFN- } \gamma \text {, IL-1 RA, } \\
\text { IL-6, IL-8, IL-10, } \\
\text { thrombin-antithrombin }\end{array}$ \\
\hline
\end{tabular}


Table 1. Cont.

\begin{tabular}{|c|c|c|c|c|c|c|c|}
\hline $\begin{array}{l}\text { Author and } \\
\text { Year }\end{array}$ & Country & Study Groups & $\begin{array}{c}\text { Sample } \\
\text { Size }(\mathrm{M} / \mathrm{F})\end{array}$ & Age (y) & $\begin{array}{c}\text { Outcome } \\
\text { Measurement Tool }\end{array}$ & Specimen & Potential Biomarkers \\
\hline $\begin{array}{l}\text { Feng et al., } \\
2020\end{array}$ & China & $\begin{array}{l}\text { Stroke rhGH group } \\
\text { Stroke placebo group }\end{array}$ & $\begin{array}{l}18 / 8 \\
17 / 9\end{array}$ & $\begin{array}{l}61.3 \pm 10 \\
60.8 \pm 11.3\end{array}$ & $\mathrm{MoCA}$ & Plasma & $\begin{array}{l}\text { TC, LDL-C, HDL-C, TG, } \\
\text { FBG, HbA1c, IGF-1, VEGF }\end{array}$ \\
\hline $\begin{array}{l}\text { Ge et al., } \\
2020\end{array}$ & China & Acute ischemic stroke & $414 / 184$ & $59.9 \pm 10.5$ & MMSE/MoCA & Serum & TIMP-1, MMP-9 \\
\hline $\begin{array}{l}\text { Gold et al., } \\
\quad 2011\end{array}$ & Canada & Ischemic stroke & $22 / 19$ & $72.3 \pm 12.2$ & MMSE & Plasma & $\begin{array}{c}\text { Tryptophan, } \\
\text { L-kynurenine, } \\
\text { L-kynurenine/tryptophan }\end{array}$ \\
\hline $\begin{array}{l}\text { Hou et al., } \\
2019\end{array}$ & China & $\begin{array}{c}\text { Total stroke } \\
\text { Stroke without PSCI } \\
\text { Stroke with PSCI }\end{array}$ & $\begin{array}{l}140 / 121 \\
65 / 55 \\
75 / 66\end{array}$ & $\begin{array}{l}66.4 \pm 9.3 \\
67.7 \pm 9.3 \\
67.7 \pm 9.3\end{array}$ & MoCA & Serum & $\begin{array}{l}\text { TC, TG, LDL-C, HDL-C, } \\
\text { hs-CRP, Hcy, retinoic acid }\end{array}$ \\
\hline $\begin{array}{l}\text { Kliper et al., } \\
2013\end{array}$ & Israel & $\begin{array}{l}\text { First-ever mild to } \\
\text { moderate stroke }\end{array}$ & & & MoCA & Serum & CRP \\
\hline $\begin{array}{l}\text { Krzystanek } \\
\text { et al., } 2007\end{array}$ & Poland & $\begin{array}{c}\text { Stroke } \\
\text { Vascular dementia } \\
\text { Alzheimer's disease }\end{array}$ & $\begin{array}{l}15 / 17 \\
13 / 19 \\
10 / 27\end{array}$ & $\begin{array}{c}74.13 \pm 7.43 \\
75.25 \pm 9.22 \\
73 \pm 6.45\end{array}$ & MMSE & Platelet & Phospholipase A2 \\
\hline $\begin{array}{l}\text { Kulesh et al., } \\
\quad 2018\end{array}$ & $\begin{array}{l}\text { Russian } \\
\text { Federation }\end{array}$ & $\begin{array}{l}\text { Normal cognition } \\
\text { Dysexecutive } \\
\text { cognitive impairment } \\
\text { Mixed cognitive } \\
\text { impairment }\end{array}$ & $\begin{array}{c}8 / 7 \\
13 / 8\end{array}$ & $\begin{array}{l}59.5 \pm 10.0 \\
66.4 \pm 8.8 \\
67.8 \pm 8.2\end{array}$ & MMSE/MoCA & Serum & IL-1 $\beta$, IL-6, IL-10, TNF $\alpha$ \\
\hline $\begin{array}{l}\text { Liu et al., } \\
2018\end{array}$ & China & $\begin{array}{l}\text { Acute ischemic stroke } \\
\text { Better outcome (mRS } \\
\text { score of } \leq 2) \\
\text { Poor outcome }(\mathrm{mRS} \\
\text { score of }>2)\end{array}$ & $\begin{array}{l}32 / 19 \\
39 / 18\end{array}$ & $\begin{array}{l}63.9 \pm 14.9 \\
66.1 \pm 16.2\end{array}$ & MMSE & Plasma & $\begin{array}{l}\text { Uric acid, creatinine, urea } \\
\mathrm{N} \text {, glucose }\end{array}$ \\
\hline $\begin{array}{l}\text { Liu et al., } \\
\quad 2017\end{array}$ & China & $\begin{array}{c}\text { Non-PSCI } \\
\text { PSCI }\end{array}$ & $\begin{array}{l}65 / 27 \\
56 / 45\end{array}$ & $\begin{array}{c}60(52.3-65.8) \\
66(56-72)\end{array}$ & MMSE & Serum & $\begin{array}{l}\text { Malondialdehyde, } \\
\text { 8-OHdG }\end{array}$ \\
\hline $\begin{array}{l}\text { Lu et al., } \\
2016\end{array}$ & China & $\begin{array}{l}\text { Acute ischemic stroke } \\
\text { Normal non-HDL-C } \\
\text { High non-HDL-C }\end{array}$ & $192 / 61$ & $\begin{array}{l}63.1 \pm 11.9 \\
62.2 \pm 10.8\end{array}$ & MMSE/MoCA & & $\begin{array}{c}\text { Non-HDL-C, TC, HDL-C, } \\
\text { LDL-C, FBG, TG, Hcy, } \\
\text { hs-CRP, HbA1c }\end{array}$ \\
\hline $\begin{array}{l}\text { Mao et al., } \\
2020\end{array}$ & China & $\begin{array}{c}\text { Non-PSCI } \\
\text { PSCI }\end{array}$ & $\begin{array}{l}79 / 37 \\
38 / 34\end{array}$ & $\begin{array}{l}65(60-74) \\
73(66-80)\end{array}$ & MoCA & Serum & $\begin{array}{c}\text { A } 342, \text { T3, T4, FT3, FT4, } \\
\text { TSH, TC, TG, HDL-C, } \\
\text { LDL-C, hs-CRP, Hcy }\end{array}$ \\
\hline $\begin{array}{l}\text { Marklund } \\
\text { et al., } 2004\end{array}$ & Sweden & Acute ischemic stroke & $169 / 99$ & $71 \pm 11$ & MMSE & Plasma/serum & $\begin{array}{c}\text { Cortisol, DS, cortisol/DS } \\
\text { ratio } \\
\text { Von Willebrand factor, } \\
\text { tissue plasminogen } \\
\text { activator, fibrinogen, } \\
\text { hs-CRP }\end{array}$ \\
\hline $\begin{array}{l}\text { Qian et al., } \\
2012\end{array}$ & China & $\begin{array}{l}\text { Stroke } \\
\text { Vascular cognitive } \\
\text { impairment with no } \\
\text { dementia } \\
\text { Vascular dementia } \\
\text { Mixed dementia }\end{array}$ & $\begin{array}{c}19 / 18 \\
18 / 18 \\
6 / 9\end{array}$ & $\begin{array}{l}62.1 \pm 1.6 \\
65.5 \pm 1.7 \\
73.8 \pm 2.1 \\
74.6 \pm 2.2\end{array}$ & MMSE/MoCA & Serum & sRAGE, BACE, neprilysin \\
\hline $\begin{array}{l}\text { Qian et al., } \\
2020\end{array}$ & China & $\begin{array}{c}\text { Endostatin } \\
\text { concentration group }\end{array}$ & $431 / 182$ & $60.0 \pm 10.5$ & MoCA & Plasma & Endostatin \\
\hline $\begin{array}{c}\text { Ran et al., } \\
2020\end{array}$ & China & $\begin{array}{l}\text { Stroke } \\
\text { PSCI }\end{array}$ & $\begin{array}{l}41 / 74 \\
43 / 39\end{array}$ & $\begin{array}{l}57.72 \pm 6.11 \\
59.99 \pm 7.46\end{array}$ & MoCA & Serum & $\begin{array}{c}\text { Uric acid, hs-CRP, } \\
\text { fibrinogen, TG, cholesterol }\end{array}$ \\
\hline $\begin{array}{l}\text { Stokowska } \\
\text { et al., } 2021\end{array}$ & Sweden & Intervention group & $64 / 51$ & & $\begin{array}{l}\text { Letter number } \\
\text { sequence test }\end{array}$ & Plasma & NfL \\
\hline $\begin{array}{l}\text { Sun et al., } \\
\quad 2020\end{array}$ & China & $\begin{array}{c}\text { Non-PSCI } \\
\text { PSCI }\end{array}$ & $\begin{array}{c}60 / 26 \\
110 / 78\end{array}$ & $\begin{array}{l}64.66 \pm 11.57 \\
71.3 \pm 10.88\end{array}$ & MoCA & Serum & $\begin{array}{c}\text { Uric acid, folic acid, VB12, } \\
\text { Hcy, TG, cholesterol, } \\
\text { HDL-C, LDL-C }\end{array}$ \\
\hline $\begin{array}{l}\text { Tang et al., } \\
2017\end{array}$ & Taiwan & $\begin{array}{c}\text { Stroke without } \\
\text { vascular dementia } \\
\text { Stroke with vascular } \\
\text { dementia }\end{array}$ & $\begin{array}{l}90 / 46 \\
21 / 15\end{array}$ & $\begin{array}{l}71.2 \pm 6.9 \\
75.4 \pm 8.8\end{array}$ & CDR/MMSE/MoCA & Plasma & sRAGE, esRAGE \\
\hline $\begin{array}{l}\text { Tong et al., } \\
2017\end{array}$ & China & $\begin{array}{c}\text { Stroke } \\
\text { Post-stroke dementia }\end{array}$ & $\begin{array}{l}21 / 21 \\
21 / 21\end{array}$ & $\begin{array}{l}75.55 \pm 2.39 \\
76.14 \pm 3.73\end{array}$ & MMSE & Plasma & $\begin{array}{l}\text { Semicarbazide-sensitive } \\
\text { amino oxidase, } \\
\text { formaldehyde }\end{array}$ \\
\hline $\begin{array}{l}\text { Wang et al., } \\
2021\end{array}$ & China & $\begin{array}{l}\text { Stable } \\
\text { Progression }\end{array}$ & $\begin{array}{c}148 / 107 \\
26 / 23\end{array}$ & $\begin{array}{l}64.86 \pm 9.37 \\
65.18 \pm 8.61\end{array}$ & MMSE/MoCA & Serum & NfL \\
\hline $\begin{array}{l}\text { Wang et al., } \\
2020\end{array}$ & China & $\begin{array}{c}\text { Control } \\
\text { Observation }\end{array}$ & $\begin{array}{l}14 / 16 \\
17 / 13\end{array}$ & $\begin{array}{l}66.1 \pm 5.9 \\
67.2 \pm 7.1\end{array}$ & MMSE/MoCA & Plasma/serum & $\begin{array}{c}\mathrm{A} \beta 40, \mathrm{~A} \beta 42, \\
\mathrm{~A} \beta 42 / \mathrm{A} \beta 40, \mathrm{CRP} \\
\text { TNF- } \alpha, \text { IL-6 }\end{array}$ \\
\hline $\begin{array}{l}\text { Wang et al., } \\
2021\end{array}$ & China & $\begin{array}{l}\text { Non-PSCI } \\
\text { PSCI }\end{array}$ & $\begin{array}{l}355 / 200 \\
538 / 491\end{array}$ & $\begin{array}{c}62 \pm 13 \\
66 \pm 18.5\end{array}$ & MoCA & Plasma & $\begin{array}{c}\text { pNfL, HbA1c, hs-CRP, } \\
\text { Hcy }\end{array}$ \\
\hline
\end{tabular}


Table 1. Cont.

\begin{tabular}{|c|c|c|c|c|c|c|c|}
\hline $\begin{array}{c}\text { Author and } \\
\text { Year }\end{array}$ & Country & Study Groups & $\begin{array}{c}\text { Sample } \\
\text { Size (M/F) }\end{array}$ & Age (y) & $\begin{array}{c}\text { Outcome } \\
\text { Measurement Tool }\end{array}$ & Specimen & Potential Biomarkers \\
\hline $\begin{array}{l}\text { Weng et al., } \\
2020\end{array}$ & China & $\begin{array}{c}\text { Non-PSCI } \\
\text { PSCI }\end{array}$ & $\begin{array}{l}130 / 67 \\
102 / 74\end{array}$ & $\begin{array}{l}64 \\
72\end{array}$ & MoCA & Blood & $\begin{array}{c}\text { CRP, TB, DBIL, IBIL, TC, } \\
\text { Ca, uric acid, HbA1c, } \\
\text { D-dimer }\end{array}$ \\
\hline $\begin{array}{l}\text { Yalbuzdag } \\
\text { et al., } 2015\end{array}$ & Turkey & $\begin{array}{c}\text { Ischemic } \\
\text { Hemorrhagic }\end{array}$ & $\begin{array}{l}53 / 43 \\
11 / 13\end{array}$ & $\begin{array}{c}63.78 \pm 12.3 \\
61.8 \pm 10.0\end{array}$ & MMSE & Plasma & $25(\mathrm{OH}) \mathrm{D}$ \\
\hline $\begin{array}{l}\text { Yan et al., } \\
2015\end{array}$ & China & $\begin{array}{c}\text { Non-vascular } \\
\text { dementia } \\
\text { Vascular dementia }\end{array}$ & $56 / 48$ & & MMSE/MoCA & Serum & Hcy, hs-CRP, LDL-C \\
\hline $\begin{array}{l}\text { Zeng et al., } \\
2019\end{array}$ & China & $\begin{array}{c}\text { Cognitive } \\
\text { impairment no } \\
\text { dementia } \\
\text { Vascular cognitive } \\
\text { impairment }\end{array}$ & $\begin{array}{l}61 / 20 \\
45 / 26\end{array}$ & $\begin{array}{l}71.40 \pm 11.32 \\
76.28 \pm 15.16\end{array}$ & MoCA & Serum & $\begin{array}{l}\text { Cystatin C, HbA1c, } \\
\text { creatinine, uric acid, TC, } \\
\text { TG, HDL-C, LDL-C }\end{array}$ \\
\hline $\begin{array}{l}\text { Zhong et al., } \\
2018\end{array}$ & China & $\begin{array}{l}\text { MMP concentration } \\
\text { group }\end{array}$ & 558 & & MMSE/MoCA & Serum & MMP-9 \\
\hline $\begin{array}{l}\text { Zhong et al., } \\
2021\end{array}$ & China & Choline/betaine/TMAO & $433 / 184$ & $60 \pm 10.5$ & MMSE/MoCA & Plasma & Choline, betaine, TMAO \\
\hline $\begin{array}{l}\text { Zhu et al., } \\
2020\end{array}$ & China & $\begin{array}{l}\text { Non-PSCI } \\
\text { PSCI }\end{array}$ & $\begin{array}{l}89 / 81 \\
50 / 36\end{array}$ & $\begin{array}{c}65 \pm 10.8 \\
71.1 \pm 10.4\end{array}$ & MMSE & Plasma & $\begin{array}{l}\text { TMAO, TC, TG, LDL-C, } \\
\text { HDL-C, hs-CRP, FBG, Hcy }\end{array}$ \\
\hline $\begin{array}{l}\text { Zhu et al., } \\
2019\end{array}$ & China & $\begin{array}{l}\text { RF concentration } \\
\text { group }\end{array}$ & 582 & & MMSE/MoCA & Serum & RF \\
\hline $\begin{array}{l}\text { Zhu et al., } \\
2019\end{array}$ & China & MMSE/MoCA group & $448 / 190$ & $60.7 \pm 10.3$ & MMSE/MoCA & Serum & $\begin{array}{c}\text { aPS, GPS, aCL, GPL, } \\
\text { ß2-GPI, RF, NT-proBNP, } \\
\text { Lp-PLA2 mass, MMP-9, } \\
\text { tHcy, eGFR, uric acid, } \\
\text { HGF }\end{array}$ \\
\hline
\end{tabular}

25(OH)D: 25-hydroxyvitamin D3, 8-OHdG: 8-hydroxydeoxyquanosine, aCL GPL: anticardiolipin antibodies, IgG anticar-diolipin antibodies units, aPS GPS: anti-phosphatidylserine antibodies, IgG antiphosphatidylserine antibodies units, A $\beta 40$ : amyloid $\beta$ 40, A $\beta 42$ : amyloid $\beta$ 42, BACE: $\beta$-secretase enzyme, BChE: butyrylcholinesterase, BDNF: brain-derived neurotrophic factor, CDR: clinical dementia rating, CRP: C-reactive protein, DBIL: direct bilirubin, DS: dehydroepiandrosterone sulphate, eGFR: estimated glomerular filtration rate, esRAGE: endogenous secretory RAGE, FBG: fasting blood glucose, FT3: free triiodothyroinine, FT4: free thyroxin, HbA1c: glycated hemoglobin, Hcy: homocysteine, HDL-C: high-density lipoprotein cholesterol, HGF: hepatocyte growth factor, hs-CRP: high-sensitivity C-reactive protein, IBIL: indirect bilirubin, IFN- $\gamma$ : interferon-gamma, IGF-1: insulin-like growth factor-1, IL-1 RA: interleukin-1 receptor antagonist, IL-10: interleukin-10, IL-1 $\beta$ : interleukin-1 beta, IL-6: interleukin-6, IL-8: interleukin-8, LDL-C: low-density lipoprotein cholesterol, Lp-PLA2: lipoprotein-associated phospholipase A2, MMP-9: matrix metalloproteinase-9, MMSE: Mini-Mental State Examination, MoCA: Montreal Cognitive Assessment, NfL: neurofilament light, NT-proBNP: N-terminal pro b-type natriuretic peptide, PSCI: post-stroke cognitive impairment, RF: rheumatoid factor, sRAGE: soluble receptor for advanced glycation end products, T3: triiodothyronine, T4: thyroxin, TB: total bilirubin, TC: total cholesterol, TG: triglyceride, tHcy: total homocysteine, TIMP-1: tissue inhibitor metalloproteinase-1, TMAO: trimethylamine N-oxide, TNF $\alpha$ : tumor necrosis factor-alpha, TSH: thyrotropin, VB12: vitamin B12, VCAM-1: vascular cell adhesion molecule 1, VEGF: vascular endothelial growth factor, $\beta 2$-GPI: beta(2)-glycoprotein 1-dependent anticardiolipin antibodies.

\subsection{Classification of Potential Blood Biomarkers for PSCI}

The potential blood biomarkers identified in the selected studies were categorized into blood and vascular functions, inflammatory and immune functions, metabolic function, neuronal function, kidney function, oxidative stress, hormones, and others (Table 2).

Of the identified potential biomarkers, the D-dimer, homocysteine (Hcy), endostatin, fibrinogen, vascular cell adhesion molecule 1 (VCAM-1), endogenous secretory receptor for advanced glycation end products (esRAGE), hs-CRP (high-sensitivity C-reactive protein) or CRP, indoleamine 2,3-dioxygenase, interleukin-10 (IL-10), interleukin-1 beta (IL-1 $\beta$ ), interleukin-6 (IL-6), kynurenine, matrix metalloproteinase-9 (MMP-9), phospholipase A2, quinolinic acid, rheumatoid factor (RF), soluble receptor for advanced glycation end products (sRAGE), semicarbazide-sensitive amino oxidase, tissue inhibitor metalloproteinase-1 (TIMP-1), trimethylamine N-oxide (TMAO), tumor necrosis factor-alpha (TNF- $\alpha$ ), kynurenine/tryptophan ratio, quinolinic acid/kynurenic acid ratio, fasting blood glucose (FBG), glycated hemoglobin (HbA1c), high-density lipoprotein cholesterol (HDL-C), low-density lipoprotein cholesterol (LDL-C), non-HDL-C, total cholesterol (TC), triglyceride (TG), $\beta$ secretase enzyme (BACE1), neprilysin, neurofilament light (NfL), cystatin $C$, uric acid, 8-hydroxydeoxyquanosine (8-OHdG), D-amino acid oxidase, malondialdehyde, N-terminal pro b-type natriuretic peptide (NT-proBNP), and cortisol levels increased among patients with PSCI. 
Table 2. Changes in the potential blood biomarkers for PSCI.

\begin{tabular}{|c|c|c|}
\hline Category & Level & Potential Biomarkers \\
\hline \multirow{2}{*}{$\begin{array}{l}\text { Blood and vascular } \\
\quad \text { functions }\end{array}$} & Increase & D-dimer, Hcy, endostatin, fibrinogen, VCAM-1 \\
\hline & No change & $\begin{array}{l}\text { Direct bilirubin, fibrinogen, Hcy, indirect bilirubin, total bilirubin, tissue plasminogen } \\
\text { activator, vitamin B12, VEGF, von Willebrand factor, thrombin-antithrombin }\end{array}$ \\
\hline \multirow[t]{3}{*}{$\begin{array}{l}\text { Inflammatory and } \\
\text { immune functions }\end{array}$} & Increase & $\begin{array}{c}\text { esRAGE, hs-CRP (CRP), indoleamine 2,3-dioxygenase, IL-10, IL-1 } \beta \text {, IL-6, kynurenine, } \\
\text { MMP-9, phospholipase A2, quinolinic acid, RF, sRAGE, semicarbazide-sensitive amino } \\
\text { oxidase, TIMP-1, TMAO, TNF- } \alpha \text {, kynurenine/tryptophan ratio, quinolinic } \\
\text { acid/kynurenic acid ratio }\end{array}$ \\
\hline & Decrease & BChE, hs-CRP (CRP), sRAGE \\
\hline & No change & $\begin{array}{l}\text { aCL GPL, aPS GPS, } \beta 2-\text { GPI, complement component 3, hs-CPR (CRP), kynurenic acid, } \\
\text { Lp-PLA2 mass, tryptophan, IFN- } \gamma \text {, IL-1 RA, IL-6, IL-8, IL-10 }\end{array}$ \\
\hline \multirow{3}{*}{ Metabolic function } & Increase & FBG, HbA1c, HDL-C, LDL-C, non-HDL-C, TC, TG \\
\hline & Decrease & Betaine, TC levels \\
\hline & No change & FBG, glucose, HbA1c, HDL-C, HGF, LDL-C, TC, TG, IGF-1 \\
\hline \multirow[t]{3}{*}{ Neuronal function } & Increase & BACE1, neprilysin, NfL \\
\hline & Decrease & BDNF, $\mathrm{A} \beta 42, \mathrm{~A} \beta 42 / \mathrm{A} \beta 40, \mathrm{NfL}$ \\
\hline & No change & S100B, A $\beta 42, A \beta 40, A C h E$, neprilysin \\
\hline \multirow[t]{3}{*}{ Kidney function } & Increase & Cystatin C, uric acid \\
\hline & Decrease & eGFR, uric acid \\
\hline & No change & Creatinine, uric acid, urea $\mathrm{N}$ \\
\hline \multirow{4}{*}{$\begin{array}{l}\text { Oxidative stress } \\
\text { Hormone }\end{array}$} & Increase & 8-OHdG, D-amino acid oxidase, malondialdehyde \\
\hline & Increase & NT-proBNP, cortisol \\
\hline & Decrease & $25(\mathrm{OH}) \mathrm{D}, \mathrm{FT} 4, \mathrm{~T} 3$ \\
\hline & No change & Cortisol/DS ratio, DS, FT3, NT-proBNP, T4, TSH \\
\hline \multirow[t]{2}{*}{ Others } & Decrease & Choline, formaldehyde, $\mathrm{NO}^{-2}$ \\
\hline & No change & $\mathrm{Ca}$, folic acid, TMAO, retinoic acid \\
\hline
\end{tabular}

25(OH)D: 25-hydroxyvitamin D3, 8-OHdG: 8-hydroxydeoxyquanosine, aCL GPL: anticardiolipin antibodies, IgG anticar-diolipin antibodies units, aPS GPS: anti-phosphatidylserine antibodies, IgG antiphosphatidylserine antibodies units, $A \beta 40$ : amyloid $\beta$ 40, A $\beta 42$ : amyloid $\beta$ 42, $\beta 2$-GPI: beta(2)-glycoprotein 1-dependent anticardiolipin antibodies, BACE: $\beta$-secretase enzyme, BChE: butyrylcholinesterase, BDNF: brain-derived neurotrophic factor, CDR: clinical dementia rating, CRP: C-reactive protein, DS: dehydroepiandrosterone sulphate, eGFR: estimated glomerular filtration rate, esRAGE: endogenous secretory RAGE, FBG: fasting blood glucose, FT3: free triiodothyroinine, FT4: free thyroxin, HbA1c: glycated hemoglobin, Hcy: homocysteine, HDL-C: high-density lipoprotein cholesterol, HGF: hepatocyte growth factor, hs-CRP: high-sensitivity C-reactive protein, IFN- $\gamma$ : interferon-gamma, IGF-1: insulin-like growth factor-1, IL-1 RA: interleukin-1 receptor antagonist, IL-10: interleukin-10, IL-1 $\beta$ : interleukin-1 beta, IL-6: interleukin-6, IL-8: interleukin-8, LDL-C: low-density lipoprotein cholesterol, Lp-PLA2: lipoprotein-associated phospholipase A2, MMP-9: matrix metalloproteinase-9, MMSE: Mini-Mental State Examination, MoCA: Montreal Cognitive Assessment, NfL: neurofilament light, NT-proBNP: N-terminal pro b-type natriuretic peptide, PSCI: post-stroke cognitive impairment, RF: rheumatoid factor, sRAGE: soluble receptor for advanced glycation end products, T3: triiodothyronine, T4: thyroxin, TC: total cholesterol, TG: triglyceride, tHcy: total homocysteine, TIMP-1: tissue inhibitor metalloproteinase-1, TMAO: trimethylamine N-oxide, TNF $\alpha$ : tumor necrosis factor-alpha, TSH: thyrotropin, VB12: vitamin B12, VCAM-1: vascular cell adhesion molecule 1, VEGF: vascular endothelial growth factor.

Meanwhile, the butyrylcholinesterase (BChE), hs-CRP or CRP, sRAGE, betaine, TC, brain-derived neurotrophic factor (BDNF), amyloid beta 42 (A $\beta 42), A \beta 42 /$ amyloid beta (A $\beta 40)$, NfL, estimated glomerular filtration rat (eGFR), uric acid, 25-hydroxyvitamin D3 $(25(\mathrm{OH}) \mathrm{D})$, free thyroxin (FT4), triiodothyronine (T3), choline, formaldehyde, and $\mathrm{NO}^{-2}$ levels decreased.

The following extracted potential blood biomarkers did not change: direct bilirubin, fibrinogen, Hcy, indirect bilirubin, total bilirubin, tissue plasminogen activator, vitamin B12, vascular endothelial growth factor, von Willebrand factor, thrombin-antithrombin, anticardiolipin antibodies, IgG anticardiolipin antibodies units (aCL GPL), anti-phosphatidylserine antibodies, IgG antiphosphatidylserine antibodies units (aPS GPS), beta(2)-glycoprotein 1-dependent anticardiolipin antibodies ( $\beta 2$-GPI), complement component 3, CRP, kynurenic acid, lipoprotein-associated phospholipase A2 mass (Lp-PLA2 mass), tryptophan, interferongamma (IFN- $\gamma$ ), interleukin-1 receptor antagonist (IL-1 RA), IL-6, IL-8, IL-10, FBG, glucose, 
HbA1c, HDL-C, hepatocyte growth factor (HGF), LDL-C, TC, TG, insulin-like growth factor 1 (IGF-1), S100B protein, A $\beta 42, \mathrm{~A} \beta 40$, acetylcholinesterase (AChE), neprilysin, creatinine, uric acid, urea $\mathrm{N}$, dehydroepiandrosterone sulphate (DS), free triiodothyroinine (FT3), NT-proBNP, thyroxin (T4), thyrotropin (TSH), Ca, folic acid, TMAO, retinoic acid, and cortisol/DS ratio.

\subsection{Meta-Analysis Results of the Hcy, hs-CRP, Uric Acid, HbA1c, TC, TG, HDL-C, and LDL-C Levels}

Figure 2 shows the meta-analysis results of the potential blood biomarkers between the PSCI and the non-PSCI group that were identified in five or more articles. As shown in Figure 2A, the Hcy level significantly differed between the PSCI and the non-PSCI groups $(\mathrm{SMD}=0.337,95 \%$ confidence interval $(\mathrm{CI})=0.100-0.573, p=0.005)$. The CRP level also significantly differed between them $(\mathrm{SMD}=0.374,95 \% \mathrm{CI}=0.121-0.628, p=0.004)$ (Figure 2B). Meanwhile, the uric acid (SMD $=0.027,95 \% \mathrm{CI}=-0.270-0.324, p=0.858$ ) (Figure 2C) and HbA1c levels (SMD $=0.036,95 \% \mathrm{CI}=-0.048-0.121, p=0.399$ ) (Figure 2D) did not significantly differ between the two groups. Furthermore, the TC level significantly differed between the PSCI and the non-PSCI groups (SMD $=0.133,95 \% \mathrm{CI}=0.022-0.244$, $p=0.019)$ (Figure 2E). The TG (SMD $=0.016,95 \% \mathrm{CI}=-0.095-0.127, p=0.777)$ (Figure 2F) and HDL-C levels (SMD $=0.198,95 \% \mathrm{CI}=-0.004-0.399, p=0.055$ ) (Figure 2G) did not significantly differ between them. As shown in Figure $2 \mathrm{H}$, the LDL-C level significantly differed between the two groups (SMD $=0.216,95 \% \mathrm{CI}=0.005-0.426, p=0.045)$.

As shown in Figure $2 \mathrm{~A}\left(I^{2}=84 \%, p<0.001\right), 2 \mathrm{~B}\left(I^{2}=87 \%, p<0.001\right), 2 \mathrm{C}\left(I^{2}=81 \%\right.$, $p=0.002), 2 \mathrm{G}\left(I^{2}=63 \%, p=0.028\right)$, and $2 \mathrm{H}\left(I^{2}=66 \%, \mathrm{p}=0.012\right)$, the heterogeneity was significant; thus, we used the random-effects model. The fixed-effects model was applied in Figure 2D $\left(I^{2}=14 \%, p=0.311\right), 2 \mathrm{E}\left(I^{2}=19 \%, p=0.291\right)$, and $2 \mathrm{~F}\left(I^{2}=44 \%\right.$, $p=0.114)$. Publication bias was evaluated using Egger's regression test. All data did not show an obvious risk of publication bias (Figure 2A: $p=0.11,2 \mathrm{~B}: p=0.10,2 \mathrm{C}: p=0.84,2 \mathrm{D}$ : $p=0.66,2 \mathrm{E}: p=0.30,2 \mathrm{~F}: p=0.76,2 \mathrm{G}: p=0.56,2 \mathrm{H}: p=0.57)$. Data that were included in this meta-analysis are shown in Supplementary Table S1.

(A) Hcy

\begin{tabular}{|c|c|c|c|c|c|c|c|c|c|c|c|c|}
\hline \multirow[t]{2}{*}{ Study name } & \multicolumn{7}{|c|}{ Statistics for each study } & \multicolumn{5}{|c|}{ Std diff in means and $95 \% \mathrm{Cl}$} \\
\hline & $\begin{array}{l}\text { Std diff } \\
\text { in means }\end{array}$ & $\begin{array}{l}\text { Standard } \\
\text { error }\end{array}$ & Variance & $\begin{array}{c}\text { Lower } \\
\text { limit }\end{array}$ & $\begin{array}{l}\text { Upper } \\
\text { limit }\end{array}$ & Z-Value & p-Value & & & & Icy & \\
\hline Hou 2019 & 0.254 & 0.125 & 0.016 & 0.010 & 0.498 & 2.036 & 0.042 & & & & & \\
\hline Mao 2020 & 0.095 & 0.150 & 0.023 & -0.200 & 0.389 & 0.631 & 0.528 & & & & & \\
\hline Sun 2020 & 0.297 & 0.131 & 0.017 & 0.041 & 0.554 & 2.274 & 0.023 & & & & & \\
\hline Wang 2021 & 0.110 & 0.034 & 0.001 & 0.045 & 0.176 & 3.291 & 0.001 & & & & & \\
\hline Yan 2015 & 1.854 & 0.323 & 0.104 & 1.221 & 2.488 & 5.737 & 0.000 & & & & + & \\
\hline \multirow[t]{4}{*}{ Zhu 2019} & 0.229 & 0.133 & 0.018 & -0.031 & 0.489 & 1.726 & 0.084 & & & & & \\
\hline & 0.337 & 0.121 & 0.015 & 0.100 & 0.573 & 2.790 & 0.005 & & & & & \\
\hline & & & & & & & & -8.00 & 4.00 & 0.00 & 4.00 & 8.00 \\
\hline & & & & & & & & & PSCI & & non-PSCI & \\
\hline
\end{tabular}

Figure 2. Cont. 


\section{(B) CRP}

\begin{tabular}{|c|c|c|c|c|c|c|c|c|c|c|c|c|}
\hline \multirow[t]{2}{*}{ Study name } & \multicolumn{7}{|c|}{ Statistics for each study } & \multicolumn{5}{|c|}{$\underline{\text { Std diff in means and } 95 \% \mathrm{Cl}}$} \\
\hline & $\begin{array}{l}\text { Std diff } \\
\text { in means }\end{array}$ & $\begin{array}{l}\text { Standard } \\
\text { error }\end{array}$ & Variance & $\begin{array}{c}\text { Lower } \\
\text { limit }\end{array}$ & $\begin{array}{c}\text { Upper } \\
\text { limit }\end{array}$ & Z-Value & p-Value & & & & $\mathbf{R P}$ & \\
\hline Choi 2020 & 1.237 & 0.414 & 0.171 & 0.425 & 2.048 & 2.987 & 0.003 & & & & | & \\
\hline Hou 2019 & 0.053 & 0.124 & 0.015 & -0.190 & 0.297 & 0.429 & 0.668 & & & & & \\
\hline Mao 2020 & 0.223 & 0.150 & 0.023 & -0.071 & 0.518 & 1.485 & 0.137 & & & & & \\
\hline $\operatorname{Ran} 2020$ & 1.127 & 0.155 & 0.024 & 0.823 & 1.432 & 7.260 & 0.000 & & & & & \\
\hline Wang 2021 & 0.048 & 0.050 & 0.002 & -0.049 & 0.146 & 0.970 & 0.332 & & & & & \\
\hline Weng 2020 & 0.287 & 0.104 & 0.011 & 0.082 & 0.491 & 2.750 & 0.006 & & & & & \\
\hline Yan 2015 & 0.139 & 0.297 & 0.088 & -0.442 & 0.721 & 0.470 & 0.639 & & & & & \\
\hline \multirow{4}{*}{ Zhu 2020} & 0.367 & 0.133 & 0.018 & 0.105 & 0.628 & 2.751 & 0.006 & & & & & \\
\hline & 0.374 & 0.129 & 0.017 & 0.121 & 0.628 & 2.895 & 0.004 & & & $1 \%$ & & \\
\hline & & & & & & & & -8.00 & 4.00 & 0.00 & 4.00 & 8.00 \\
\hline & & & & & & & & & PSCI & & on-PSCI & \\
\hline
\end{tabular}

\section{(C) Uric acid}

\begin{tabular}{|c|c|c|c|c|c|c|c|c|c|c|c|c|}
\hline \multirow[t]{2}{*}{ Study name } & \multicolumn{7}{|c|}{ Statistics for each study } & \multicolumn{5}{|c|}{ Std diff in means and $95 \% \mathrm{Cl}$} \\
\hline & $\begin{array}{l}\text { Std diff } \\
\text { in means }\end{array}$ & $\begin{array}{l}\text { Standard } \\
\text { error }\end{array}$ & Variance & $\begin{array}{l}\text { Lower } \\
\text { limit }\end{array}$ & $\begin{array}{l}\text { Upper } \\
\text { limit }\end{array}$ & Z-Value & p-Value & \multicolumn{5}{|c|}{ Uric acid } \\
\hline $\operatorname{Ran} 2020$ & -0.341 & 0.146 & 0.021 & -0.626 & -0.056 & -2.342 & 0.019 & & & & . & I \\
\hline Sun 2020 & 0.372 & 0.131 & 0.017 & 0.115 & 0.629 & 2.835 & 0.005 & & & & & \\
\hline Weng 2020 & -0.091 & 0.104 & 0.011 & -0.294 & 0.113 & -0.873 & 0.383 & & & & & \\
\hline \multirow[t]{4}{*}{ Zeng 2019} & 0.175 & 0.163 & 0.027 & -0.144 & 0.495 & 1.076 & 0.282 & & & & & \\
\hline & 0.027 & 0.151 & 0.023 & -0.270 & 0.324 & 0.179 & 0.858 & & & & & \\
\hline & & & & & & & & -8.00 & -4.00 & 0.00 & 4.00 & 8.00 \\
\hline & & & & & & & & & PSCI & & non-PSCI & \\
\hline
\end{tabular}

(D) $\mathrm{HbA} 1 \mathrm{c}$

\begin{tabular}{|c|c|c|c|c|c|c|c|c|c|c|c|c|c|}
\hline \multirow[t]{2}{*}{ Model } & \multirow[t]{2}{*}{ Study name } & \multicolumn{7}{|c|}{ Statistics for each study } & \multicolumn{5}{|c|}{ Std diff in means and $95 \% \mathrm{Cl}$} \\
\hline & & $\begin{array}{l}\text { Std diff } \\
\text { in means }\end{array}$ & $\begin{array}{l}\text { Standard } \\
\text { error }\end{array}$ & Variance & $\begin{array}{c}\text { Lower } \\
\text { limit }\end{array}$ & $\begin{array}{c}\text { Upper } \\
\text { limit }\end{array}$ & Z-Value & $\mathrm{p}$-Value & & & HbA1 & & \\
\hline & Wang 2021 & 0.006 & 0.050 & 0.002 & -0.091 & 0.104 & 0.124 & 0.901 & & 1 & & | & 1 \\
\hline & Weng 2020 & 0.181 & 0.104 & 0.011 & -0.023 & 0.385 & 1.740 & 0.082 & & & 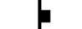 & & \\
\hline & Zeng 2019 & 0.006 & 0.163 & 0.026 & -0.312 & 0.325 & 0.038 & 0.969 & & & & & \\
\hline \multirow[t]{3}{*}{ Fixed } & & 0.036 & 0.043 & 0.002 & -0.048 & 0.121 & 0.843 & 0.399 & & & & & \\
\hline & & & & & & & & & -8.00 & -4.00 & 0.00 & 4.00 & 8.00 \\
\hline & & & & & & & & & & $\mathrm{PSCl}$ & & non-PSCI & \\
\hline
\end{tabular}

(E) TC

\begin{tabular}{|c|c|c|c|c|c|c|c|c|c|c|c|c|c|}
\hline \multirow[t]{2}{*}{ Model } & \multirow[t]{2}{*}{ Study name } & \multicolumn{7}{|c|}{ Statistics for each study } & \multicolumn{5}{|c|}{ Std diff in means and $95 \% \mathrm{Cl}$} \\
\hline & & $\begin{array}{l}\text { Std diff } \\
\text { in means }\end{array}$ & $\begin{array}{l}\text { Standard } \\
\text { error }\end{array}$ & Variance & $\begin{array}{l}\text { Lower } \\
\text { limit }\end{array}$ & $\begin{array}{c}\text { Upper } \\
\text { limit }\end{array}$ & Z-Value & $p$-Value & & & TC & & \\
\hline & Hou 2019 & 0.190 & 0.124 & 0.015 & -0.054 & 0.434 & 1.523 & 0.128 & & 1 & & & 1 \\
\hline & Mao 2020 & 0.054 & 0.150 & 0.023 & -0.240 & 0.348 & 0.359 & 0.720 & & & & & \\
\hline & $\operatorname{Ran} 2020$ & 0.071 & 0.145 & 0.021 & -0.212 & 0.354 & 0.491 & 0.623 & & & & & \\
\hline & Sun 2020 & 0.094 & 0.130 & 0.017 & -0.161 & 0.350 & 0.724 & 0.469 & & & & & \\
\hline & Zeng 2019 & 0.479 & 0.165 & 0.027 & 0.156 & 0.802 & 2.904 & 0.004 & & & & & \\
\hline & Zhu 2020 & 0.000 & 0.132 & 0.018 & -0.259 & 0.259 & 0.000 & 1.000 & & & & & \\
\hline \multirow[t]{3}{*}{ Fixed } & & 0.133 & 0.057 & 0.003 & 0.022 & 0.244 & 2.341 & 0.019 & & & & & \\
\hline & & & & & & & & & -8.00 & -4.00 & 0.00 & 4.00 & 8.00 \\
\hline & & & & & & & & & & $\mathrm{PSCl}$ & & non-PSCI & \\
\hline
\end{tabular}

Figure 2. Cont. 
(F) TG

\begin{tabular}{|c|c|c|c|c|c|c|c|c|c|c|c|c|c|}
\hline \multirow[t]{2}{*}{ Model } & \multirow[t]{2}{*}{ Study name } & \multicolumn{7}{|c|}{ Statistics for each study } & \multicolumn{5}{|c|}{ Std diff in means and $95 \% \mathrm{Cl}$} \\
\hline & & $\begin{array}{l}\text { Std diff } \\
\text { in means }\end{array}$ & $\begin{array}{l}\text { Standard } \\
\text { error }\end{array}$ & Variance & $\begin{array}{c}\text { Lower } \\
\text { limit }\end{array}$ & $\begin{array}{c}\text { Upper } \\
\text { limit }\end{array}$ & $Z$-Value & p-Value & & & TG & & \\
\hline & Hou 2019 & 0.081 & 0.124 & 0.015 & -0.163 & 0.324 & 0.652 & 0.515 & & I & & 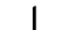 & | \\
\hline & Mao 2020 & 0.356 & 0.151 & 0.023 & 0.060 & 0.652 & 2.356 & 0.018 & & & & & \\
\hline & $\operatorname{Ran} 2020$ & -0.073 & 0.145 & 0.021 & -0.356 & 0.211 & -0.502 & 0.616 & & & & & \\
\hline & Sun 2020 & -0.202 & 0.130 & 0.017 & -0.457 & 0.054 & -1.545 & 0.122 & & & & & \\
\hline & Zeng 2019 & -0.080 & 0.163 & 0.026 & -0.399 & 0.238 & -0.494 & 0.621 & & & & & \\
\hline & Zhu 2020 & 0.044 & 0.132 & 0.018 & -0.216 & 0.303 & 0.331 & 0.741 & & & & & \\
\hline \multirow[t]{3}{*}{ Fixed } & & 0.016 & 0.057 & 0.003 & -0.095 & 0.127 & 0.283 & 0.777 & & & & & \\
\hline & & & & & & & & & -8.00 & -4.00 & 0.00 & 4.00 & 8.00 \\
\hline & & & & & & & & & & PSCI & & non-PSC & \\
\hline
\end{tabular}

(G) HDL-C

\begin{tabular}{|c|c|c|c|c|c|c|c|c|c|c|c|c|}
\hline \multirow[t]{2}{*}{ Study name } & \multicolumn{7}{|c|}{ Statistics for each study } & \multicolumn{5}{|c|}{ Std diff in means and $95 \% \mathrm{Cl}$} \\
\hline & $\begin{array}{l}\text { Std diff } \\
\text { in means }\end{array}$ & $\begin{array}{l}\text { Standard } \\
\text { error }\end{array}$ & Variance & $\begin{array}{c}\text { Lower } \\
\text { limit }\end{array}$ & $\begin{array}{l}\text { Upper } \\
\text { limit }\end{array}$ & z-Value & $\mathrm{p}$-Value & & & HDL-C & & \\
\hline Hou 2019 & 0.000 & 0.124 & 0.015 & -0.243 & 0.243 & 0.000 & 1.000 & & & & | & \\
\hline Mao 2020 & 0.346 & 0.151 & 0.023 & 0.050 & 0.642 & 2.289 & 0.022 & & & & & \\
\hline Sun 2020 & 0.000 & 0.130 & 0.017 & -0.255 & 0.255 & 0.000 & 1.000 & & & & & \\
\hline Zeng 2019 & 0.164 & 0.163 & 0.027 & -0.155 & 0.483 & 1.009 & 0.313 & & & & & \\
\hline \multirow[t]{4}{*}{ Zhu 2019} & 0.500 & 0.134 & 0.018 & 0.237 & 0.763 & 3.727 & 0.000 & & & & & \\
\hline & 0.198 & 0.103 & 0.011 & -0.004 & 0.399 & 1.919 & 0.055 & & & & & \\
\hline & & & & & & & & -8.00 & -4.00 & 0.00 & 4.00 & 8.00 \\
\hline & & & & & & & & & $\mathrm{PSCl}$ & & non-PSCl & \\
\hline
\end{tabular}

(H) LDL-C

\begin{tabular}{|c|c|c|c|c|c|c|c|c|c|c|c|c|}
\hline \multirow[t]{2}{*}{ Study name } & \multicolumn{7}{|c|}{ Statistics for each study } & \multicolumn{5}{|c|}{ Std diff in means and $95 \% \mathrm{Cl}$} \\
\hline & $\begin{array}{c}\text { Std diff } \\
\text { in means }\end{array}$ & $\begin{array}{l}\text { Standard } \\
\text { error }\end{array}$ & Variance & $\begin{array}{c}\text { Lower } \\
\text { limit }\end{array}$ & $\begin{array}{c}\text { Upper } \\
\text { limit }\end{array}$ & z-Value & $\mathrm{p}$-Value & & & LDL-C & & \\
\hline Hou 2019 & 0.035 & 0.124 & 0.015 & -0.209 & 0.278 & 0.280 & 0.780 & & & & 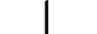 & $\mid$ \\
\hline Mao 2020 & 0.688 & 0.154 & 0.024 & 0.386 & 0.990 & 4.462 & 0.000 & & & & & \\
\hline Sun 2020 & 0.012 & 0.130 & 0.017 & -0.243 & 0.267 & 0.090 & 0.928 & & & & & \\
\hline Yan 2015 & 0.168 & 0.297 & 0.088 & -0.413 & 0.750 & 0.567 & 0.571 & & & & & \\
\hline Zeng 2019 & 0.303 & 0.164 & 0.027 & -0.017 & 0.624 & 1.854 & 0.064 & & & & & \\
\hline \multirow[t]{4}{*}{ Zhu 2020} & 0.125 & 0.132 & 0.018 & -0.134 & 0.385 & 0.947 & 0.344 & & & & & \\
\hline & 0.216 & 0.108 & 0.012 & 0.005 & 0.426 & 2.002 & 0.045 & & & & & \\
\hline & & & & & & & & -8.00 & -4.00 & 0.00 & 4.00 & 8.00 \\
\hline & & & & & & & & & $\mathrm{PSCl}$ & & non-PSCl & \\
\hline
\end{tabular}

Figure 2. Forest plots of the (A) Hcy, (B) CRP, (C) uric acid, (D) HbA1c, (E) TC, (F) TG, (G) HDL-C, and (H) LDL-C levels. Hcy: homocysteine, CRP: C-reactive protein, HbA1c: glycated hemoglobin, TC: total cholesterol, TG: triglyceride, HDL-C: high-density lipoprotein cholesterol, LDL-C: lowdensity lipoprotein cholesterol, Std diff: standard difference, CI: confidence interval, PSCI: post-stroke cognitive impairment.

\section{Discussion}

Recent studies have highlighted the potential of blood-derived parameters as biomarkers for timely patient triage, therapeutics, and stroke mechanisms [30-32]. Easily accessible fluid biomarkers can provide an objective evaluation of the real-time panorama, supporting stroke diagnosis or predicting the patients' outcome and ultimately guiding clinical decisions [20,32-35]. Cognitive impairment tends to progressively worsen following stroke, with $20-30 \%$ of patients developing dementia [9,36]. Hence, international guidelines recommend cognitive assessment as a routine neurological examination for all stroke survivors [37]. Cognitive function refers to mental processes that are crucial for conducting 
activities of daily living. Such mental processes include attention, short-term and long-term memory, reasoning, coordination of movement, and planning of tasks [38]. The prevalence of brain disorders affecting cognition, such as stroke and dementia, increases steadily in a linear fashion with age [39]. Therefore, we evaluated the levels of eight proteins in the peripheral blood as biomarkers for stroke, especially related to brain damage and cognitive impairments.

First, we investigated whether the levels of four plasma lipids could be potential biomarkers for PSCI individually. As shown in Figure 2, the plasma levels of both TC and LDL-C were higher in the PSCI group than in the non-PSCI group. However, there was no difference in the HDL-C and TG levels between them. In fact, disorders of lipid homeostasis are common risk factors for cardiovascular diseases, which are linked to Alzheimer's disease (AD) (Dement, 2016). Abnormal levels of lipids or lipoproteins in the blood, which include high levels of LDL-C and low levels of HDL-C, TC, and TG, were related to the cause of the disease outbreak $[40,41]$. Cholesterol, an important constituent of mammalian cell membranes, modulates membrane fluidity and permeability. It is also the precursor of all steroid hormones and bile acids and plays a key role in membrane trafficking, transmembrane signaling processes, and cell proliferation [42-46]. Cholesterol is typically transported as lipoproteins, which consist of TG and cholesterol in the center surrounded by a phospholipid shell with apolipoprotein embedded in them $[47,48]$. The relationship between lipids and cognition is controversial. Peripheral cholesterol levels have been evaluated in some studies [13,49-52]; Kumral et al., (2015). Most studies did not find any association between cholesterol levels and PSCI [13,50-52]; however, two studies found a significant association. One study showed that higher levels of LDL-C and lower levels of HDL-C were independently associated with PSCI [53]. The TG levels were studied in only four studies [49,51-53]. One study found an association between the baseline TG levels and PSCI [49,54]. Nevertheless, in the central nervous system (CNS), altered levels of cholesterol appear to be involved in several neurodegenerative diseases, such as AD, Niemann-Pick C disease, and major depressive disorder [55-57]. In addition, cholesterol-driven inflammation seems to affect neuroplasticity, altering membrane fluidity and permeability, vesicular trafficking and monoamine release, and neuroendocrine function [57-61]. The possibility that the differentiation and function of the CNS were partly influenced by the level of LDL-C or HDL-C circulating in the plasma received further support from recent developments in our understanding of the molecular events involved in transmembrane cholesterol movement [62]. Therefore, we insist that the TC and LDL-C levels could be potential biomarkers for PSCI, and understanding the prognostic impact of these levels in relation to PSCI may be clinically relevant. Although our results on the HDL-C and TG levels were not significant, it is necessary to consider them in future research.

Second, the levels of Hcy were higher in the PSCI group than in the non-PSCI group, as shown in Figure 2. Hcy is produced in all cells and is involved in the metabolism of cysteine and methionine [63]. In previous cross-sectional and prospective studies, elevated plasma Hcy levels have been associated with more than 100 diseases, syndromes, or outcomes [64]. Hcy metabolism is largely dependent on B vitamins, including folate, vitamin B12, and vitamin B6. Substantial lowering of the Hcy level can be achieved through B vitamin supplementation, suggesting a safe and inexpensive intervention strategy for reducing age-related cognitive decline and the risk of AD and overall dementia [65]. Whether Hcy contributes to cerebrovascular changes, cognitive function, or both remains controversial; nevertheless, it appears that elevated Hcy levels are a risk factor for dementia in older adults, regardless of the mechanism. The relationship between the Hcy levels and cognition is controversial. Although there were negative results that suggest that the Hcy levels were not associated with cognitive decline after stroke $[49,66]$, it is a known fact that a high Hcy level is an independent risk factor for cerebrovascular events $[67,68]$ and cognitive impairment [69-72]. In addition, a recent study showed that patients with higher Hcy levels had greater cortical and hippocampal atrophy than those with lower levels [73]. 
Therefore, the Hcy level could be a potential biomarker for PSCI, and understanding its prognostic impact in relation to PSCI may be clinically relevant.

Third, the levels of CRP were higher in the PSCI group than in the non-PSCI group (Figure 2). CRP is a plasma protein synthesized by the liver and is often used as a nonspecific biomarker of inflammation. Elevated CRP levels are associated with an increased risk of cerebrovascular diseases and dementia [24]. Upregulation of CRP is considered a marker of systemic inflammation in autoimmune conditions, infection, and obesity [74]. Thus, CRP plays dual roles as a marker of inflammation and a driver of the induction of inflammation [75]. There is evidence that it infiltrates the brain following BBB disruption by hemorrhagic or ischemic stroke [76-78]. CRP in ischemic tissues drives the progression of $\mathrm{AD}$ following ischemic stroke [75,79]. The relationship between the CRP level and cognition is controversial. Inconsistent results on the link between specific cognitive functions and proinflammatory markers have been reported. For example, the CRP levels have been demonstrated to be negatively related to performance in tests of episodic memory and to be predictive of poorer memory performance in some studies [80,81], but not in all $[82,83]$. Nevertheless, increased CRP levels may cause cognitive impairment. A previous study has found an independent association between the CRP levels and PSCI [84]. In addition, the CRP levels were negatively associated with a composite score of executive function and processing speed [85]. Therefore, the CRP level could be a potential biomarker for PSCI, and understanding its prognostic impact in relation to PSCI may be clinically relevant.

Fourth, there was no difference in the HbA1c and uric acid levels between the PSCI and non-PSCI groups. The relationship between the $\mathrm{HbA1c}$ levels and cognition is controversial. The HbA1c levels were evaluated in some studies. One study showed no significant association with PSCI [13]; another study showed an independent association between higher $\mathrm{HbA1c}$ levels and PSCI [86]. Further, there has been an accumulation of studies investigating the link between the uric acid levels and neurodegenerative diseases, mainly including dementia, Parkinson's disease, amyotrophic lateral sclerosis, and multiple system atrophy [87]. A study of cognitively healthy adults found that elevated baseline serum uric acid levels were associated with decreased attention and visuospatial abilities in men [88]. Although our results on the $\mathrm{HbA1c}$ and uric acid levels were not significant, it is necessary that these levels be considered in future research.

In addition to the eight proteins selected in our study, we demonstrated that many other proteins could be considered as possible biomarkers; however, we could not metaanalyze these proteins because of insufficient data, as shown in Table 2. Many potential biomarkers for PSCI have been suggested. (1) The levels of the blood and vascular-functionrelated proteins, such as D-dimer, endostatin, fibrinogen, and VCAM-1, were higher in patients with PSCI than in those without; (2) the levels of the inflammatory and immunefunction-related proteins, such as esRAGE, indoleamine 2,3-dioxygenase, IL-10, IL-1 IL-6, kynurenine, kynurenine/tryptophan ratio, MMP-9, phospholipase A2, quinolinic acid, quinolinic acid/kynurenic acid ratio, RF, sRAGE, SSAO, TIMP-1, TMAO, TNF- $\alpha$, and $\mathrm{BChE}$, significantly differed between patients with PSCI and those without; (3) the levels of the metabolic-function-related proteins, such as FBG, non-HDL-C, and betaine, significantly differed between them; (4) the levels of the neuronal-function-related proteins, such as BACE1, neprilysin, NfL, BDNF, A $\beta 42$, and A $\beta 42 / A \beta 40$, also significantly differed between them; (5) the levels of the kidney-function-related proteins, such as cystatin $C$ and epidermal growth factor receptor, also significantly differed between them; (6) the levels of the oxidative-stress-related proteins, such as 8-OHdG, D-amino acid oxidase, and malondialdehyde, were higher in patients with PSCI than in patients with stroke only; (7) the levels of the hormones, such as NT-proBNP, cortisol, 25(OH)D, FT4, and T3, significantly differed between patients with PSCI and those without; and (8) the levels of the other compounds, such as choline, formaldehyde, and $\mathrm{NO}^{-2}$, were lower in patients with PSCI than in those without. Nevertheless, further research on these candidate biomarkers is needed to validate, identify, and introduce useful biomarkers for stroke recurrence or diagnosis in a scalable manner in medical practice [17]. 
This study had certain limitations. Although potential blood biomarkers for PSCI were collected in various study groups, the meta-analysis was performed using available data between the PSCI and the non-PSCI group. Furthermore, this study did not consider the characteristics of the patient groups, such as the stage and duration of stroke. It has been addressed that the meta-analysis itself has poor quality of included studies, heterogeneity, and publication bias [89]. However, our study is obviously meaningful in selecting new potential biomarkers for PSCI because meta-analysis can be conceived as a systematic study of all studies that have been conducted to answer a specific question or hypothesis [90]. Our study also included patients after stroke onset, regardless of comparison with other neurodegenerative diseases. Therefore, further research is needed to compare patients with stroke with patients with mild cognitive impairment in the early stage of cognitive decline or with patients with other neurodegenerative diseases.

Nevertheless, our study demonstrated that the levels of some proteins, including TC, LDL-C, Hcy, and CRP, remarkably changed in patients with post-stroke cognitive impairment. Therefore, some blood-derived proteins, such as TC, LDL-C, Hcy, and CRP, could be potential biomarkers for the diagnosis, prognosis prediction, and progression evaluation of stroke, especially related to brain damage and cognitive impairments.

\section{Materials and Methods}

\subsection{Literature Search and Selection Criteria}

This systematic review was performed in accordance with the Preferred Reporting Items for Systematic Reviews and Meta-Analyses guidelines. All publications that described the association between PSCI and human blood biomarkers were searched in PubMed, Embase, the Web of Science, and the Cochrane Library from inception to 26 July 2021. In PubMed, we used the following search terms: (stroke OR stroke [Mesh] OR "cerebral infarct" OR "brain infarct" OR "cerebral hemorrhage" OR "cerebral hemorrhage" OR "cerebral ischemia" OR "cerebral ischemia" OR "cerebral hematoma" OR "cerebral hematoma" OR "brain hemorrhage" OR "brain hemorrhage") AND (dementia OR dementia [Mesh] OR "cognitive decline" OR "cognitive impairment" OR cognition disorder OR cognition disorders [Mesh]) AND (biomarker OR biomarker [Mesh]) AND (blood OR serum OR plasma). In Embase, the Web of Science, and the Cochrane Library, the following terms were used: (stroke OR "cerebral infarct" OR "brain infarct" OR "cerebral hemorrhage" OR "cerebral hemorrhage" OR "cerebral ischemia" OR "cerebral ischemia" OR “cerebral hematoma" OR "cerebral hematoma" OR "brain hemorrhage" OR "brain hemorrhage") AND (dementia OR "cognitive decline" OR "cognitive impairment" OR "cognition disorder") AND (biomarker) AND (blood OR serum OR plasma). The inclusion criteria were as follows: (1) articles that included patients with cognitive impairment or dementia after stroke; and (2) articles that identified blood biomarkers for cognitive function after stroke.

\subsection{Data Extraction and Analysis}

Two authors (K.Y. Kim and K.-A. Chang) independently screened and selected relevant studies according to the inclusion criteria. Any disagreements on every step were resolved via constant discussion with all authors. The following relevant data were extracted from the 40 selected studies: article information, including the title, name of first author, year of publication, and country of patients; group information, including the types of group, sample size, sex, age, and measurement tool used for evaluating cognitive function; and biomarker information, including the type of specimen and type and level of potential biomarkers. For the meta-analysis, the standardized mean difference (SMD) in the potential biomarkers for evaluating PSCI was analyzed between patients with and without PSCI using the Comprehensive Meta-Analysis software version 3 (Biostats Inc., Englewood, NJ, USA). A fixed- or random-effects model was used after analyzing the Q statistic, while the $I^{2}$ method was applied to assess heterogeneity. Funnel plots and Egger's intercept tests 
were used to evaluate publication bias. The statistical significance level was set at $p$-values of $<0.05$.

Supplementary Materials: The following are available online at https:/ /www.mdpi.com/article/10 .3390/ijms23020602/s1.

Author Contributions: Conceptualization, K.-A.C. and K.Y.K.; formal analysis, K.-A.C. and K.Y.K.; investigation, K.Y.S.; writing-original draft, K.Y.S. and K.Y.K.; writing-review and editing, K.Y.S. and K.-A.C.; supervision, K.-A.C.; project administration, K.-A.C.; funding acquisition, K.-A.C. All authors have read and agreed to the published version of the manuscript.

Funding: This research was supported by the Bio \& Medical Technology Development Program of the National Research Foundation (NRF) funded by the Korean government (MSIT) (2020M3A9E4104384) and by the Basic Science Research Program through the NRF of Korea funded by the Ministry of Education (2020R1I1A1A01070793). The funders had no role in the study design, data collection and analysis, decision to publish, or preparation of the manuscript.

Institutional Review Board Statement: Not applicable.

Informed Consent Statement: Not applicable.

Data Availability Statement: The data that support the findings of this study are available from the corresponding authors upon reasonable request.

Conflicts of Interest: The authors declare no conflict of interest.

\section{References}

1. Krishnamurthi, R.V.; Ikeda, T.; Feigin, V.L. Global, regional and country-specific burden of ischaemic stroke, intracerebral haemorrhage and subarachnoid haemorrhage: A systematic analysis of the global burden of disease study 2017. Neuroepidemiology 2020, 54, 171-179. [CrossRef]

2. Jia, J.; Zhang, H.; Liang, X.; Dai, Y.; Liu, L.; Tan, K.; Ma, R.; Luo, J.; Ding, Y.; Ke, C. Application of metabolomics to the discovery of biomarkers for ischemic stroke in the murine model: A comparison with the clinical results. Mol. Neurobiol. 2021. [CrossRef]

3. Campbell, B.C.V.; Khatri, P. Stroke. Lancet 2020, 396, 129-142. [CrossRef]

4. Zhang, L.F.; Yang, J.; Hong, Z.; Yuan, G.G.; Zhou, B.F.; Zhao, L.C.; Huang, Y.N.; Chen, J.; Wu, Y.F.; Collaborative Group of China Multicenter Study of Cardiovascular, E. Proportion of different subtypes of stroke in China. Stroke 2003, 34, 2091-2096. [CrossRef] [PubMed]

5. Woodruff, T.M.; Thundyil, J.; Tang, S.C.; Sobey, C.G.; Taylor, S.M.; Arumugam, T.V. Pathophysiology, treatment, and animal and cellular models of human ischemic stroke. Mol. Neurodegener. 2011, 6, 11. [CrossRef]

6. Zhang, P.; Wang, C.; Wu, J.; Zhang, S. A systematic review of the predictive value of plasma D-dimer levels for Predicting stroke outcome. Front. Neurol. 2021, 12, 693524. [CrossRef]

7. Grotta, J.C.; Albers, G.W.; Broderick, J.P.; Day, A.L.; Kasner, S.E.; Lo, E.H.; Sacco, R.L.; Wong, L.K.S. Stroke: Pathophysiology, Diagnosis, and Management, 7th. ed.; Elsevier, Inc.: Philadelphia, PA, USA, 2021.

8. Fride, Y.; Adamit, T.; Maeir, A.; Ben Assayag, E.; Bornstein, N.M.; Korczyn, A.D.; Katz, N. What are the correlates of cognition and participation to return to work after first ever mild stroke? Top Stroke Rehabil 2015, 22, 317-325. [CrossRef] [PubMed]

9. Mijajlovic, M.D.; Pavlovic, A.; Brainin, M.; Heiss, W.D.; Quinn, T.J.; Ihle-Hansen, H.B.; Hermann, D.M.; Assayag, E.B.; Richard, E.; Thiel, A.; et al. Post-stroke dementia-A comprehensive review. BMC Med. 2017, 15, 11. [CrossRef]

10. Onyike, C.U. Cerebrovascular disease and dementia. Int. Rev. Psychiatry. 2006, 18, 423-431. [CrossRef] [PubMed]

11. Kalaria, R.N.; Akinyemi, R.; Ihara, M. Stroke injury, cognitive impairment and vascular dementia. Biochim. Biophys. Acta. 2016, 1862, 915-925. [CrossRef]

12. Vataja, R.; Pohjasvaara, T.; Mantyla, R.; Ylikoski, R.; Leppavuori, A.; Leskela, M.; Kalska, H.; Hietanen, M.; Aronen, H.J.; Salonen, O.; et al. MRI correlates of executive dysfunction in patients with ischaemic stroke. Eur. J. Neurol. 2003, 10, 625-631. [CrossRef]

13. Kandiah, N.; Wiryasaputra, L.; Narasimhalu, K.; Karandikar, A.; Marmin, M.; Chua, E.V.; Sitoh, Y.Y. Frontal subcortical ischemia is crucial for post stroke cognitive impairment. J. Neurol. Sci. 2011, 309, 92-95. [CrossRef] [PubMed]

14. Stephens, S.; Kenny, R.A.; Rowan, E.; Allan, L.; Kalaria, R.N.; Bradbury, M.; Ballard, C.G. Neuropsychological characteristics of mild vascular cognitive impairment and dementia after stroke. Int. J. Geriatr. Psychiatry 2004, 19, 1053-1057. [CrossRef] [PubMed]

15. Roman, G.C.; Tatemichi, T.K.; Erkinjuntti, T.; Cummings, J.L.; Masdeu, J.C.; Garcia, J.H.; Amaducci, L.; Orgogozo, J.M.; Brun, A.; Hofman, A.; et al. Vascular dementia-Diagnostic-criteria for research studies-Report of the Ninds-Airen international workshop. Neurology 1993, 43, 250-260. [CrossRef] [PubMed]

16. Schnider, A.; Regard, M.; Landis, T. Anterograde and retrograde amnesia following bitemporal infarction. Behav. Neurol. 1994, 7, 87-92. [CrossRef] 
17. Baez, S.D.; del Barco, D.G.; Hardy-Sosa, A.; Nieto, G.G.; Bringas-Vega, M.L.; Llibre-Guerra, J.J.; Valdes-Sosa, P. Scalable Bio marker combinations for early stroke diagnosis: A systematic review. Front. Neurol. 2021, 12. [CrossRef]

18. Maas, M.B.; Furie, K.L. Molecular biomarkers in stroke diagnosis and prognosis. Biomark. Med. 2009, 3, 363-383. [CrossRef]

19. Alex Matos Ribeiro, J.; Fernanda Garcia-Salazar, L.; Regina Saade-Pacheco, C.; Shirley Moreira Silva, E.; Garcia Oliveira, S.; Flavia Silveira, A.; Sanches Garcia-Araujo, A.; Luiz Russo, T. Prognostic molecular markers for motor recovery in acute hemorrhagic stroke: A systematic review. Clin. Chim. Acta 2021, 522, 45-60. [CrossRef]

20. Biomarkers Definitions Working, Group. Biomarkers and surrogate endpoints: Preferred definitions and conceptual framework. Clin. Pharmacol. Ther. 2001, 69, 89-95. [CrossRef]

21. Andone, S.; Bajko, Z.; Motataianu, A.; Mosora, O.; Balasa, R. The role of biomarkers in atherothrombotic stroke-A systematic review. Int. J. Mol. Sci. 2021, 22. [CrossRef]

22. Boyd, L.A.; Hayward, K.S.; Ward, N.S.; Stinear, C.M.; Rosso, C.; Fisher, R.J.; Carter, A.R.; Leff, A.P.; Copland, D.A.; Carey, L.M.; et al. Biomarkers of stroke Recovery: Consensus-based core recommendations from the stroke recovery and rehabilitation roundtable. Neurorehabil. Neural Repair 2017, 31, 864-876. [CrossRef] [PubMed]

23. Troiani, Z.; Ascanio, L.; Rossitto, C.P.; Ali, M.; Mohammadi, N.; Majidi, S.; Mocco, J.; Kellner, C.P. Prognostic utility of serum biomarkers in intracerebral hemorrhage: A systematic review. Neurorehabil. Neural Repair 2021, 15459683211041314. [CrossRef] [PubMed]

24. Zhang, X.; Bi, X. Post-stroke cognitive impairment: A review focusing on molecular biomarkers. J. Mol. Neurosci. 2020, 70, 1244-1254. [CrossRef] [PubMed]

25. Verdelho, A.; Wardlaw, J.; Pavlovic, A.; Pantoni, L.; Godefroy, O.; Duering, M.; Charidimou, A.; Chabriat, H.; Biessels, G.J. Cognitive impairment in patients with cerebrovascular disease: A white paper from the ESO Dementia Committee. Eur. Stroke J. 2021, 6, 5-17. [CrossRef]

26. Kulesh, A.; Drobakha, V.; Kuklina, E.; Nekrasova, I.; Shestakov, V. Cytokine response, tract-specific fractional anisotropy, and brain morphometry in post-stroke cognitive impairment. J. Stroke Cerebrovasc. Dis. 2018, 27, 1752-1759. [CrossRef]

27. Ball, E.L.; Sutherland, R.; Squires, C.; Mead, G.E.; Religa, D.; Lundstrom, E.; Cheyne, J.; Wardlaw, J.M.; Quinn, T.J.; Shenkin, S.D. Predicting post-stroke cognitive impairment using acute CT neuroimaging: A systematic review and meta-analysis. Int. J. Stroke 2021, 17474930211045836. [CrossRef] [PubMed]

28. Lim, J.S.; Lee, J.J.; Woo, C.W. Post-stroke cognitive impairment: Pathophysiological insights into brain disconnectome from advanced neuroimaging analysis techniques. J. Stroke 2021, 23, 297-311. [CrossRef]

29. Potter, T.; Lioutas, V.A.; Tano, M.; Pan, A.; Meeks, J.; Woo, D.; Seshadri, S.; Selim, M.; Vahidy, F. Cognitive impairment after intracerebral Hemorrhage: A systematic review of current evidence and knowledge gaps. Front. Neurol. 2021, $12,716632$. [CrossRef]

30. Kalra, L.P.; Khatter, H.; Ramanathan, S.; Sapehia, S.; Devi, K.; Kaliyaperumal, A.; Bal, D.; Sebastian, I.; Kakarla, R.; Singhania, A.; et al. Serum GFAP for stroke diagnosis in regions with limited access to brain imaging (BE FAST India). Eur. Stroke J. 2021, 6, 176-184. [CrossRef]

31. Bustamante, A.; Penalba, A.; Orset, C.; Azurmendi, L.; Llombart, V.; Simats, A.; Pecharroman, E.; Ventura, O.; Ribo, M.; Vivien, D.; et al. Blood biomarkers to differentiate Ischemic and hemorrhagic strokes. Neurology 2021, 96, e1928-e1939. [CrossRef]

32. Dias, A.; Silva, I.; Pinto, I.M.; Maia, L.F. Timely and blood-based multiplex molecular profiling of acute stroke. Life 2021, 11, 816. [CrossRef]

33. Park, D.; Joo, S.S.; Lee, H.J.; Choi, K.C.; Kim, S.U.; Kim, Y.B. Microtubule-associated protein 2, an early blood marker of ischemic brain injury. J. Neurosci. Res. 2012, 90, 461-467. [CrossRef]

34. Misra, S.; Kumar, A.; Kumar, P.; Yadav, A.K.; Mohania, D.; Pandit, A.K.; Prasad, K.; Vibha, D. Blood-based protein biomarkers for stroke differentiation: A systematic review. Proteomics Clin. Appl. 2017, 11. [CrossRef]

35. Wang, L.; Song, Q.; Wang, C.; Wu, S.; Deng, L.; Li, Y.; Zheng, L.; Liu, M. Neutrophil to lymphocyte ratio predicts poor outcomes after acute ischemic stroke: A cohort study and systematic review. J. Neurol. Sci. 2019, 406, 116445. [CrossRef]

36. Pendlebury, S.T.; Rothwell, P.M. Prevalence, incidence, and factors associated with pre-stroke and post-stroke dementia: A systematic review and meta-analysis. Lancet Neurol. 2009, 8, 1006-1018. [CrossRef]

37. Hachinski, V.; Iadecola, C.; Petersen, R.C.; Breteler, M.M.; Nyenhuis, D.L.; Black, S.E.; Powers, W.J.; DeCarli, C.; Merino, J.G.; Kalaria, R.N.; et al. National Institute of Neurological Disorders and Stroke-Canadian Stroke Network vascular cognitive impairment harmonization standards. Stroke 2006, 37, 2220-2241. [CrossRef]

38. de Champlain, J.; Wu, R.; Girouard, H.; Karas, M.A.; El Midaoui, A.; Laplante, M.A.; Wu, L. Oxidative stress in hypertension. Clin. Exp. Hypertens. 2004, 26, 593-601. [CrossRef] [PubMed]

39. Verhaeghen, P.; Salthouse, T.A. Meta-analyses of age-cognition relations in adulthood: Estimates of linear and nonlinear age effects and structural models. Psychol. Bull. 1997, 122, 231-249. [CrossRef] [PubMed]

40. Alzheimer's, Association. 2016 Alzheimer's disease facts and figures. Alzheimers Dement. 2016, 12, 459-509. [CrossRef] [PubMed]

41. Liu, Y.; Zhong, X.; Shen, J.; Jiao, L.; Tong, J.; Zhao, W.; Du, K.; Gong, S.; Liu, M.; Wei, M. Elevated serum TC and LDL-C levels in Alzheimer's disease and mild cognitive impairment: A meta-analysis study. Brain Res. 2020, 1727, 146554. [CrossRef]

42. Fernandez, C.; Lobo, M.D.V.T.; Gomez-Coronado, D.; Lasuncion, M.A. Cholesterol is essential for mitosis progression and its deficiency induces polyploid cell formation. Exp. Cell Res. 2004, 300, 109-120. [CrossRef] [PubMed] 
43. Fernandez, C.; Martin, M.; Gomez-Coronado, D.; Lasuncion, M.A. Effects of distal cholesterol biosynthesis inhibitors on cell proliferation and cell cycle progression. J. Lipid Res. 2005, 46, 920-929. [CrossRef] [PubMed]

44. Hooper, N.M. Detergent-insoluble glycosphingolipid/cholesterol-rich membrane domains, lipid rafts and caveolae. Mol. Membr. Biol. 1999, 16, 145-156. [CrossRef]

45. Nwokoro, N.A.; Wassif, C.A.; Porter, F.D. Genetic disorders of cholesterol biosynthesis in mice and humans. Mol. Genet. Metab. 2001, 74, 105-119. [CrossRef] [PubMed]

46. Goedeke, L.; Fernandez-Hernando, C. Regulation of cholesterol homeostasis. Cell Mol. Life Sci. 2012, 69, 915-930. [CrossRef]

47. Ohvo-Rekila, H.; Ramstedt, B.; Leppimaki, P.; Slotte, J.P. Cholesterol interactions with phospholipids in membranes. Prog. Lipid Res. 2002, 41, 66-97. [CrossRef]

48. Laudanski, K. Persistence of lipoproteins and cholesterol alterations after sepsis: Implication for atherosclerosis progression. Int. J. Mol. Sci. 2021, 22, 517. [CrossRef]

49. Barba, R.; Martinez-Espinosa, S.; Rodriguez-Garcia, E.; Pondal, M.; Vivancos, J.; Del Ser, T. Poststroke dementia: Clinical features and risk factors. Stroke 2000, 31, 1494-1501. [CrossRef]

50. Rasquin, S.M.; Verhey, F.R.; van Oostenbrugge, R.J.; Lousberg, R.; Lodder, J. Demographic and CT scan features related to cognitive impairment in the first year after stroke. J. Neurol. Neurosurg. Psychiatry 2004, 75, 1562-1567. [CrossRef]

51. Baum, L.; Chen, X.; Cheung, W.S.; Cheung, C.K.; Cheung, L.W.; Chiu, K.F.; Wen, H.M.; Poon, P.; Woo, K.S.; Ng, H.K.; et al Polymorphisms and vascular cognitive impairment after ischemic stroke. J. Geriatr. Psychiatry Neurol. 2007, 20, 93-99. [CrossRef]

52. Tamam, B.; Tasdemir, N.; Tamam, Y. The prevalence of dementia three months after stroke and its risk factors. Turk. Psikiyatri. Derg. 2008, 19, 46-56.

53. Kumral, E.; Gulluoglu, H.; Alakbarova, N.; Deveci, E.E.; Colak, A.Y.; Caginda, A.D.; Evyapan, D.; Orman, M. Cognitive decline in patients with leukoaraiosis within 5 years after initial stroke. J. Stroke Cerebrovasc. Dis. 2015, 24, 2338-2347. [CrossRef] [PubMed]

54. Casolla, B.; Caparros, F.; Cordonnier, C.; Bombois, S.; Henon, H.; Bordet, R.; Orzi, F.; Leys, D. Biological and imaging predictors of cognitive impairment after stroke: A systematic review. J. Neurology 2019, 266, 2593-2604. [CrossRef] [PubMed]

55. Ikonen, E. Mechanisms for cellular cholesterol transport: Defects and human disease. Physiol. Rev. 2006, 86, 1237-1261. [CrossRef] [PubMed]

56. Maxfield, F.R.; Tabas, I. Role of cholesterol and lipid organization in disease. Nature 2005, 438, 612-621. [CrossRef]

57. Gliozzi, M.; Musolino, V.; Bosco, F.; Scicchitano, M.; Scarano, F.; Nucera, S.; Zito, M.C.; Ruga, S.; Carresi, C.; Macri, R.; et al Cholesterol homeostasis: Researching a dialogue between the brain and peripheral tissues. Pharmacol. Res. 2021, 163. [CrossRef]

58. Quan, G.; Xie, C.; Dietschy, J.M.; Turley, S.D. Ontogenesis and regulation of cholesterol metabolism in the central nervous system of the mouse. Brain Res. Dev. Brain Res. 2003, 146, 87-98. [CrossRef]

59. Saher, G.; Brugger, B.; Lappe-Siefke, C.; Mobius, W.; Tozawa, R.; Wehr, M.C.; Wieland, F.; Ishibashi, S.; Nave, K.A. High cholesterol level is essential for myelin membrane growth. Nat. Neurosci. 2005, 8, 468-475. [CrossRef]

60. Morell, P.; Jurevics, H. Origin of cholesterol in myelin. Neurochem. Res. 1996, 21, 463-470. [CrossRef]

61. Maiuolo, J.; Maretta, A.; Gliozzi, M.; Musolino, V.; Carresi, C.; Bosco, F.; Mollace, R.; Scarano, F.; Palma, E.; Scicchitano, M.; et al. Ethanol-induced cardiomyocyte toxicity implicit autophagy and NFkB transcription factor. Pharmacol. Res. 2018, 133, 141-150. [CrossRef]

62. Dietschy, J.M.; Turley, S.D. Thematic review series: Brain lipids. Cholesterol metabolism in the central nervous system during early development and in the mature animal. J. Lipid Res. 2004, 45, 1375-1397. [CrossRef]

63. Selhub, J. Homocysteine metabolism. Annu. Rev. Nutr. 1999, 19, 217-246. [CrossRef]

64. Smith, A.D.; Refsum, H. Homocysteine-From disease biomarker to disease prevention. J. Intern. Med. 2021, 290, 826-854. [CrossRef]

65. McCaddon, A.; Miller, J.W. Assessing the association between homocysteine and cognition: Reflections on Bradford Hill, meta-analyses, and causality. Nutr. Rev. 2015, 73, 723-735. [CrossRef] [PubMed]

66. Sachdev, P.S.; Brodaty, H.; Valenzuela, M.J.; Lorentz, L.; Looi, J.C.; Berman, K.; Ross, A.; Wen, W.; Zagami, A.S. Clinical determinants of dementia and mild cognitive impairment following ischaemic stroke: The Sydney stroke study. Dement. Geriatr. Cogn. Disord. 2006, 21, 275-283. [CrossRef] [PubMed]

67. Hassan, A.; Hunt, B.J.; O'Sullivan, M.; Bell, R.; D'Souza, R.; Jeffery, S.; Bamford, J.M.; Markus, H.S. Homocysteine is a risk factor for cerebral small vessel disease, acting via endothelial dysfunction. Brain 2004, 127, 212-219. [CrossRef] [PubMed]

68. Ostrakhovitch, E.A.; Tabibzadeh, S. Homocysteine and age-associated disorders. Ageing Res. Rev. 2019, 49, 144-164. [CrossRef]

69. Garcia, A.; Zanibbi, K. Homocysteine and cognitive function in elderly people. Can. Med. Assoc. J. 2004, 171, 897-904. [CrossRef]

70. Price, B.R.; Wilcock, D.M.; Weekman, E.M. Hyperhomocysteinemia as a risk factor for vascular contributions to cognitive impairment and dementia. Front. Aging Neurosci. 2018, 10. [CrossRef]

71. Sala, I.; Sanchez-Saudinos, M.B.; Molina-Porcel, L.; Lazaro, E.; Gich, I.; Clarimon, J.; Blanco-Vaca, F.; Blesa, R.; Gomez-Isla, T.; Lleo, A. Homocysteine and cognitive impairment relation with diagnosis and neuropsychological performance. Dement. Geriatr. Cogn. Disord. 2008, 26, 506-512. [CrossRef]

72. Zhou, S.; Chen, J.; Cheng, L.; Fan, K.; Xu, M.; Ren, W.; Chen, Y.; Geng, D.; Cheng, H.; Luan, X.; et al. Age-dependent association between elevated homocysteine and cognitive impairment in a post-stroke population: A prospective study. Front. Nutr. 2021, 8 , 691837. [CrossRef] 
73. Den Heijer, T.; Vermeer, S.E.; Clarke, R.; Oudkerk, M.; Koudstaal, P.J.; Hofman, A.; Breteler, M.M. Homocysteine and brain atrophy on MRI of non-demented elderly. Brain 2003, 126, 170-175. [CrossRef]

74. Hsuchou, H.; Kastin, A.J.; Mishra, P.K.; Pan, W. C-reactive protein increases BBB permeability: Implications for obesity and neuroinflammation. Cell Physiol. Biochem. 2012, 30, 1109-1119. [CrossRef] [PubMed]

75. Jeon, M.T.; Kim, K.S.; Kim, E.S.; Lee, S.; Kim, J.; Hoe, H.S.; Kim, D.G. Emerging pathogenic role of peripheral blood factors following BBB disruption in neurodegenerative disease. Ageing Res. Rev. 2021, 68, 101333. [CrossRef] [PubMed]

76. Di Napoli, M.; Godoy, D.A.; Campi, V.; Masotti, L.; Smith, C.J.; Parry Jones, A.R.; Hopkins, S.J.; Slevin, M.; Papa, F.; Mogoanta, L.; et al. C-reactive protein in intracerebral hemorrhage: Time course, tissue localization, and prognosis. Neurology 2012, 79, 690-699. [CrossRef]

77. Di Napoli, M.; Parry-Jones, A.R.; Smith, C.J.; Hopkins, S.J.; Slevin, M.; Masotti, L.; Campi, V.; Singh, P.; Papa, F.; Popa-Wagner, A.; et al. C-reactive protein predicts hematoma growth in intracerebral hemorrhage. Stroke 2014, 45, 59-65. [CrossRef] [PubMed]

78. Di Napoli, M.; Slevin, M.; Popa-Wagner, A.; Singh, P.; Lattanzi, S.; Divani, A.A. Monomeric C-reactive protein and cerebral Hemorrhage: From bench to bedside. Front. Immunol. 2018, 9, 1921. [CrossRef]

79. Slevin, M.; Matou, S.; Zeinolabediny, Y.; Corpas, R.; Weston, R.; Liu, D.; Boras, E.; Di Napoli, M.; Petcu, E.; Sarroca, S.; et al. Monomeric C-reactive protein-A key molecule driving development of Alzheimer's disease associated with brain ischaemia? Sci. Rep. 2015, 5, 13281. [CrossRef]

80. Komulainen, P.; Lakka, T.A.; Kivipelto, M.; Hassinen, M.; Penttila, I.M.; Helkala, E.L.; Gylling, H.; Nissinen, A.; Rauramaa, R. Serum high sensitivity C-reactive protein and cognitive function in elderly women. Age Ageing 2007, 36, 443-448. [CrossRef]

81. Bettcher, B.M.; Wilheim, R.; Rigby, T.; Green, R.; Miller, J.W.; Racine, C.A.; Yaffe, K.; Miller, B.L.; Kramer, J.H. C-reactive protein is related to memory and medial temporal brain volume in older adults. Brain Behav. Immun. 2012, 26, 103-108. [CrossRef]

82. Hoth, K.F.; Haley, A.P.; Gunstad, J.; Paul, R.H.; Poppas, A.; Jefferson, A.L.; Tate, D.F.; Ono, M.; Jerskey, B.A.; Cohen, R.A. Elevated C-reactive protein is related to cognitive decline in older adults with cardiovascular disease. J. Am. Geriatr. Soc. 2008, 56, 1898-1903. [CrossRef] [PubMed]

83. Wersching, H.; Duning, T.; Lohmann, H.; Mohammadi, S.; Stehling, C.; Fobker, M.; Conty, M.; Minnerup, J.; Ringelstein, E.B.; Berger, K.; et al. Serum C-reactive protein is linked to cerebral microstructural integrity and cognitive function. Neurology 2010, 74, 1022-1029. [CrossRef] [PubMed]

84. Guo, J.; Su, W.; Fang, J.H.; Chen, N.; Zhou, M.K.; Zhang, Y.; He, L. Elevated CRP at admission predicts post-stroke cognitive impairment in Han Chinese patients with intracranial arterial stenosis. Neurol. Res. 2018, 40, 292-296. [CrossRef]

85. Tegeler, C.; O'Sullivan, J.L.; Bucholtz, N.; Goldeck, D.; Pawelec, G.; Steinhagen-Thiessen, E.; Demuth, I. The inflammatory markers CRP, IL-6, and IL-10 are associated with cognitive function—data from the Berlin aging study II. Neurobiol. Aging 2016, 38, 112-117. [CrossRef] [PubMed]

86. Ben Assayag, E.; Eldor, R.; Korczyn, A.D.; Kliper, E.; Shenhar-Tsarfaty, S.; Tene, O.; Molad, J.; Shapira, I.; Berliner, S.; Volfson, V.; et al. Type 2 diabetes mellitus and impaired renal function are associated with brain alterations and poststroke cognitive decline. Stroke 2017, 48, 2368-2374. [CrossRef]

87. Qiao, M.; Chen, C.; Liang, Y.; Luo, Y.; Wu, W. The influence of serum uric acid level on Alzheimer's Disease: A narrative review. BioMed. Res. Int. 2021, 2021, 5525710. [CrossRef]

88. Kueider, A.M.; An, Y.; Tanaka, T.; Kitner-Triolo, M.H.; Studenski, S.; Ferrucci, L.; Thambisetty, M. Sex-dependent associations of serum uric acid with brain function during aging. J. Alzheimers Dis. 2017, 60, 699-706. [CrossRef]

89. Noble, J.H., Jr. Meta-analysis: Methods, strengths, weaknesses, and political uses. J. Lab. Clin. Med. 2006, 147, 7-20. [CrossRef]

90. Esterhuizen, T.M.; Thabane, L. Con: Meta-analysis: Some key limitations and potential solutions. Nephrol. Dial. Transplant. 2016, 31, 882-885. [CrossRef] [PubMed] 